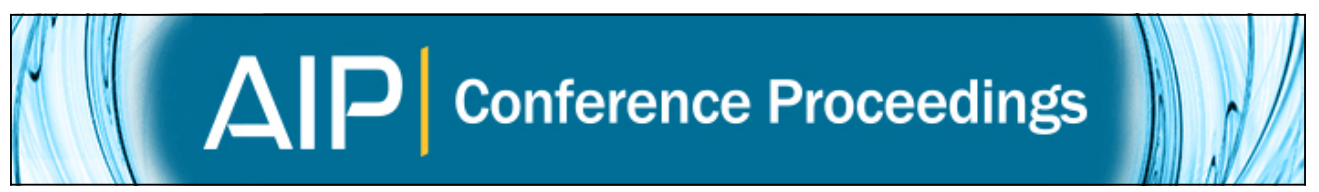

A self-consistent approach to the reflection component in 4U 1705-44

A. D'Aì, T. Di Salvo, R. laria, A. Papitto, and D. Ballantyne

Citation: AIP Conference Proceedings 1248, 63 (2010); doi: 10.1063/1.3475355

View online: http://dx.doi.org/10.1063/1.3475355

View Table of Contents:

http://scitation.aip.org/content/aip/proceeding/aipcp/1248?ver=pdfcov

Published by the AIP Publishing

Articles you may be interested in

The self-consistent nonlinear theory of electron cyclotron maser based on anomalous Doppler effect

Appl. Phys. Lett. 98, 261502 (2011); 10.1063/1.3604016

Self-consistent interaction of neutrals and shocks in the local interstellar medium

AlP Conf. Proc. 719, 323 (2004); 10.1063/1.1809536

Self-Consistent Modelling of Polychromatic ICRH in Tokamaks

AIP Conf. Proc. 694, 126 (2003); 10.1063/1.1638010

Dust scattered X-ray halo of 4 U 1538-52 observed with ASCA

AIP Conf. Proc. 599, 794 (2001); 10.1063/1.1434744

BeppoSAX observation of the X-ray pulsar 4U 1538-52

AIP Conf. Proc. 510, 213 (2000); 10.1063/1.1303205 


\title{
A self-consistent approach to the reflection component in $4 \mathrm{U}$ 1705-44
}

\author{
A. D’Aï, T. Di Salvo*, R. Iaria*, A. Papitto ${ }^{\dagger}$ and D. Ballantyne** \\ *DSFA, Universitá di Palermo, via Archirafi n.36, 90123 Palermo, Italy. \\ ${ }^{\dagger}$ Università degli Studi di Cagliari, Dipartimento di Fisica, SP Monserrato-Sestu, KM 0.7, 09042 \\ Monserrato, Italy \\ ${ }^{* *}$ Center for Relativistic Astrophysics, Georgia Tech, USA
}

\begin{abstract}
High-resolution spectroscopy has recently revealed in many neutron-star Low-Mass Xray binaries that the shape of the broad iron line observed in the $6.4-6.97 \mathrm{keV}$ range is consistently well fitted by a relativistically smeared line profile. We show here spectral fitting results using a newly developed self-consistent reflection model on XMM-Newton data of the LMXB 4U 1705-44 during a period when the source was in a bright soft state. This reflection model adopts a blackbody prescription for the shape of the impinging radiation field, that we physically associate with the boundary layer emission.
\end{abstract}

Keywords: accretion discs - stars: individual: 4U 1705-44 — stars: neutron stars — X-ray: stars - X-ray: spectrum - X-ray: general

PACS: $97.80 . J p$

\section{INTRODUCTION}

$4 \mathrm{U} 1705-44$ is a persistently bright accreting neutron-star Low-Mass X-ray Binary (LMXB) located in the direction of the Galactic Bulge. 4U 1705-44 shows a secular trend of alternating high- and low-flux periods on a variable timescale of the order of months. Spectral analysis with the Chandra high resolution gratings revealed, superimposed to the continuum, a set of local features, the most prominent of which was the fluorescent iron line [1]. Following these results, an XMM-Newton observation in August 2008 provided evidence that the broad emission lines in the source spectrum is due to the combined Keplerian and relativistic effects of disk reflecting matter at few gravitational radii from the compact object [2]. We re-analyze these XMM-Newton data in order to show that the reflection component can be fitted using a self-consistent model (refbb). In this work, we use the reflection table model described in Ballantyne [3]. This reflection model is calculated for an optically thick atmosphere, irradiated by a black-body incident spectrum of $\mathrm{kT}_{I O N}$ temperature. The model gives the reflected spectrum according to the ionization parameter $\xi$, and the relative abundance of iron with respect to the other metals.

\section{SPECTRAL ANALYSIS AND MODELS}

XMM-Newton observed 4U 1705-44 on 2008 August 24th from 02:57:04 to 17:13:35 UTC (Obs. ID 0551270201), for a duration of $51390 \mathrm{~s}$ and an effective exposure of 
$45170 \mathrm{~s}$. For spectral analysis we used RGS1 data in the 1.0-2.0 keV energy range and EPIC/PN data in the 2.0-12.0 keV range. We used standard $S A S$ tools (version 9.0.0) for data selection, masks generation, ancillary and response matrices. Spectra were filtered according to the PATTERN $\leq 4$ and $F L A G=0$ criteria. Background spectra above $12 \mathrm{keV}$ were inspected to check for the presence of periods of high solar background Background spectra have been extracted using same filtering criteria of source spectra in the RAWX 3-10 columns. Source spectra were extracted in the RAWX 28-46, but excluding the brightest row in order to avoid pile-up effects in our spectral analysis. We grouped the EPIC/pn data in order to have five energy channels per resolution element and at least 25 counts per energy channel. This choice avoids an excessive oversampling of the data, without loss of important spectral information. RGS data were also grouped adopting the 25 counts per energy channel criterion. For spectral fitting we used Xspec version 12.5.0. The interstellar absorption (phabs component in Xspec) is calculated using the cross-sections table of Verner et al. [4] and the metal abundances table of Wilms et al. [5]. The best-fitting value of the $\mathrm{N}_{\mathrm{H}}$ is $2.70 \pm 0.22 \times 10^{22} \mathrm{~cm}^{-2}$. The source was in a very soft state during the observation and the spectrum can be well described by the sum of two thermal components: a soft $(\mathrm{kT} \sim 1 \mathrm{keV})$ multicolor disk emission and a harder component, that we simply model using a blackbody spectrum. Replacing the harder blackbody component with a thermal Comptonization [2] has no effect on the chi square of the best fit and on the parameter determination of the other spectral components, so that our choice to adopt the blackbody component for the hard part of the spectrum is fully consistent.

The disk temperature is $1.15 \pm 0.03 \mathrm{keV}$, while the derived inner disk radius (assuming a distance from the source of $7.4 \mathrm{kpc}$ and an inclination of the disk of $35^{\circ}$ ) is 11.3 $\pm 0.5 \mathrm{~km}$. The harder blackbody temperature is $1.91_{-0.06}^{+0.20} \mathrm{keV}$ and the corresponding blackbody radius is $5.3 \pm 0.2 \mathrm{~km}$. To model broad positive residuals, we used the reflection model refbb, convolved with the rablur component [6] to take into account the relativistic (in a Schwarzschild space-time) and Doppler effects of the disk irradiated matter. The degree of smearing is set by the disk inner and outer radius (in units of gravitational radii $\mathrm{R}_{g}=G M / c^{2} \sim 2 \mathrm{~km}$ for a 1.4 solar mass $\mathrm{NS}$ ), the inclination angle of the disk and the emissivity law profile, assumed to only depend from the distance of the source of the impinging photons $\left(\varepsilon(r)=r^{-q}\right)$. These parameters are well constrained by the fitting, except for the disk outer radius, that we choose to keep frozen at $10^{4}$ $\mathrm{R}_{g}$, given its much larger uncertainty with respect to the other spectral parameters. We added to this model two other zero-width Gaussians (smeared using the same rdblur component) at $3.32 \mathrm{keV}$ and at $3.90 \mathrm{keV}$ corresponding to the Lyman- $\alpha$ transitions of $\mathrm{Ar}$ XVIII and Ca XIX, because of still evident residuals at these energies and the absence of the corresponding resonant transitions for argon and calcium in the relection model that we adopted. The reflection component is also well constrained and the broad iron line is consistently well fitted, leaving no other residual in the iron range (see Fig. 1). The ionization parameter $\log \xi$ is $2.36_{-0.18}^{+0.33}$, indicating a strongly ionized reflecting surface, consistent with the association of the iron line with a resonant transition of He-like iron in Di Salvo et al. [2]. The temperature of the irradiating blackbody spectrum, $\mathrm{kT}_{I O N}$, is $1.9 \pm 0.1 \mathrm{keV}$ and is compatible with the temperature of the hard blackbody continuum component, suggesting a physical and natural link between these two temperatures.

This model gives a reduced $\chi^{2}$ of 1.3 (706 dof) and the residuals do not show any 


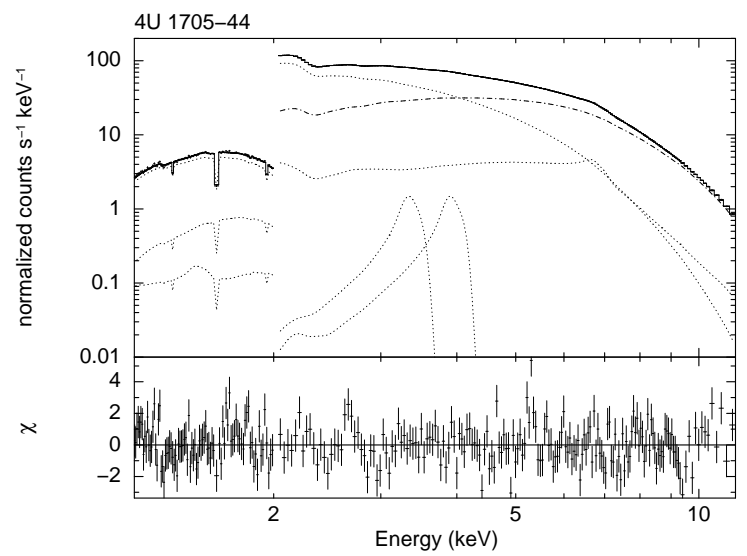

FIGURE 1. XMM-Newton data together with the best-fitting model (upper panel). Data rebinned for clarity. In lower panel the residuals with respect to the best-fitting model in units of $\sigma$.
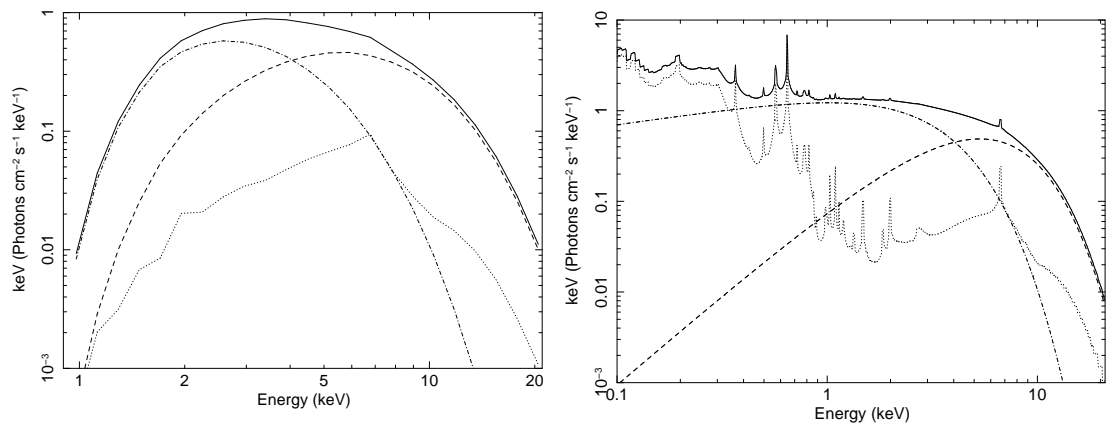

FIGURE 2. The theretical model in the $E \times f(E)$ representation. The left panel shows the spectrum when absorbed by the interstellar medium and with the blurring component applied to the reflection component; the right panel shows the theoretical unabsorbed spectrum and the unblurred reflection component. Solid, dash, dash-dot and dot-dot lines indicate the total, hard blackbody, thermal disk and reflection spectrum, respectively.

other local feature to be present. The blurring parameters are very well constrained: the inner radius is $<13 \mathrm{R}_{g}$, the system inclination is $35 \pm 2$ degrees, and the $q$ parameter is $-2.3 \pm 0.1$. In Fig. 1, we show the data, together with the best-fitting model, while in Fig. 2 we show the theoretical model in the $E \times f(E)$ representation.

The observed 1.0-10.0 keV absorbed flux in this observation is $5.58 \times 10^{-9} \mathrm{erg} \mathrm{s}^{-1}$, while the unabsorbed flux is $1.16 \times 10^{-8} \mathrm{erg} \mathrm{s}^{-1}$. Extrapolation of this model from 0.1 $\mathrm{keV}$ to $100 \mathrm{keV}$ results in an unabsorbed flux of $1.63 \times 10^{-8} \mathrm{erg} \mathrm{s}^{-1}$. The inferred X-ray luminosity in this state corresponds to $\sim 1 \times 10^{38} \mathrm{erg} \mathrm{s}^{-1}$. 


\section{CONCLUSIONS}

During the observation 4U 170544 was in a luminous, soft state. The luminosity of the source was at $\sim 50 \%$ of the Eddington limit, with most of the X-ray emission within the observed 1-12 keV X-ray band ( $\sim 70 \%)$. The spectrum is adequatly well fitted by the sum of two thermal components together with a reflection component. If we associate the blackbody hard emission with the boundary layer (BL) emission, this model accounts for the energetics and spectral distribution between the different continuum components. The BL should occupy a very small region between the inner disk radius and the surface of the NS and it is the expected site for the Comptonization of seed photons from the disk and the surface of the NS in a hot optically thick corona in these high-accretion states [7]. The thickness of the corona is such to Compton-saturate the seed photon field, and a blackbody spectrum is thus resulting.

The disk and the BL have comparable fluxes, with a luminosity ratio $L_{B L} / L_{d i s k}$ of 1.67 (assuming a spherical isotropic emission for the BL, and neglecting the reflection contribution) or 2.0 (with the reflection emission summed to the hard component).

The reflection component in this state is remarkably strong and the reprocessed flux at the disk surface is about $30 \%$ of the Comptonized component flux. The reflection component, although most clearly determined by the shape of the broad iron line, gives its most important contribution in the very soft X-ray band. Below $1 \mathrm{keV}$ there is $62 \%$ of the overall flux of the component, with a strong contribution from the of $\mathrm{H}$ - and He-like carbon, oxygen and neon resonant lines (see Fig. 2, left panel). In the 1-10 keV range, the relative contribution is $33 \%$, while above $10 \mathrm{keV}$, there is only $5 \%$ flux, due to the very soft nature of the incident flux. We calculated the reflection fraction $(R=\Omega / 2 \pi)$ for this model to be $0.23 \pm 0.01$, a value that is consistent with our proposed geometry of a compact illuminating source.

\section{REFERENCES}

1. T. Di Salvo, R. Iaria, M. Méndez, L. Burderi, G. Lavagetto, N. R. Robba, L. Stella, and M. van der Klis, ApJ 623, L121-L124 (2005), arXiv: astro-ph/ 0503224.

2. T. Di Salvo, A. D'Ai', R. Iaria, L. Burderi, M. Dovčiak, V. Karas, G. Matt, A. Papitto, S. Piraino, A. Riggio, N. R. Robba, and A. Santangelo, ArXiv e-prints (2009), 0904.3318.

3. D. R. Ballantyne, MNRAS 351, 57-62 (2004), arXiv : astro-ph/ 0402603.

4. D. A. Verner, G. J. Ferland, K. T. Korista, and D. G. Yakovlev, ApJ 465, 487-+ (1996), arXiv : astro-ph/9601009.

5. J. Wilms, A. Allen, and R. McCray, ApJ 542, 914-924 (2000), arXiv: astro-ph/ 0008425.

6. A. C. Fabian, M. J. Rees, L. Stella, and N. E. White, MNRAS 238, 729-736 (1989).

7. R. Popham, and R. Sunyaev, ApJ 547, 355-383 (2001). 